\title{
Upper airway obstruction and raised intracranial pressure in children with craniosynostosis
}

\author{
S. Gonsalez*, R. Hayward**, B. Jones**, R. Lane*
}

Upper airway obstruction and raised intracranial pressure in children with craniosynostosis. S. Gonsalez, R. Hayward, B. Jones, R. Lane. (CERS Journals Ltd 1997. ABSTRACT: In children with craniosynostosis, raised intracranial pressure (ICP) and upper airway obstruction (UAO) are both common features. However, potential interactions between UAO and ICP during sleep are poorly understood. The aim of the present study was to compare the levels of ICP during sleep between a group of patients with syndromic craniosynostosis (with facial involvement and consequent UAO) and a group of control patients with isolated unicoronal synostosis (with no facial involvement and normal upper airways).

Polygraphic cardiorespiratory sleep studies with continuous monitoring of ICP were performed during unsedated sleep in 13 children with syndromic craniosynostosis and 7 control patients with isolated unicoronal synostosis only.

In the syndromic group, UAO was present in 11 out of 13 patients, with 8 out of 13 having frank obstructive sleep apnoea. In contrast, none of the control patients showed signs of UAO during sleep. There was no evidence of central apnoeas in any of the patients studied. Clinical histories taken from parents tended to underestimate the severity of the respiratory problems. Elevated ICP was seen in 10 of the 13 syndromic patients, with borderline raised ICP in the remaining three cases. In contrast, raised ICP was seen in only 3 of the 7 control patients, with borderline raised levels in 2 of the 7. For both patient groups, ICP was higher during active sleep compared to quiet sleep. Multiple regression analysis showed that ICP during active sleep was dependent upon disease severity (unicoronal/multiple synostosis) and to the baseline ICP level during quiet sleep. Both raised ICP and airway obstruction were more apparent during active sleep. There was a significant correlation between severity of UAO and increased ICP in active sleep.

We conclude that obstructive respiratory problems are frequent in the syndromic patients, and can be severe in a large proportion of cases; intracranial hypertension is also frequent in this group. Further studies are required to investigate the possibility of a causal relationship between upper airway obstruction and raised intracranial pressure.

Eur Respir J 1997; 10: 367-375.

*Portex Anaesthesia, Intensive Therapy \& Respiratory Medicine Unit, and **Craniofacial Unit, Institute of Child Health and Great Ormond Street Hospital for Children NHS Trust, University of London, UK.

Correspondence: S. Gonsalez Portex Anaesthesia

Intensive Therapy \& Respiratory Medicine Unit

Institute of Child Health and Great Ormond Street Hospital for Children NHS Trust

30 Guilford Street

London WC1N 1EH

UK

Keywords: Child

craniosynostosis

intracranial pressure

obstructive sleep apnoea

Received: March 141996

Accepted after revision October 241996

Craniosynostosis consists of the premature closure of one or more of the skull sutures. Primary craniosynostosis affects approximately 1 in 1,000 infants [1]. It can manifest itself as an isolated feature, which is commonly sporadic, with the involvement of only one suture, or, alternatively, the condition can present as part of a syndrome, in which case two or more sutures are usually affected. The anatomical problems associated with craniosynostosis can be broadly divided into two groups: 1) deformities of the cranium; and 2) midfacial (maxillary) hypoplasia.

The abnormal skull morphology varies in form and severity. The consequences of cranial deformities are well-recognized and are primarily: altered cosmetic appearance, restricted brain growth; and intracranial hypertension [2]. Whilst reference values for intracranial pressure (ICP) in normal children cannot be obtained, in craniosynostosis a mean ICP up to $10 \mathrm{mmHg}$ has been considered as probably normal, $10-15 \mathrm{mmHg}$ borderline, with intracranial hypertension defined as above $15 \mathrm{mmHg}$ [3].

Midfacial hypoplasia is a common feature of many of the craniosynostosis syndromes $[4,5]$. Beside the more obvious cosmetic problems, midfacial hypoplasia may also have important functional implications as a result of morphological abnormalities of the nasal, oral and pharyngeal airways. In these patients, both feeding and breathing can be impaired to various degrees.

From the respiratory point of view, upper airway obstruction (UAO) is frequently observed clinically, and it can vary from mild obstruction to severe obstructive sleep apnoea (OSA). It is now recognized that severe upper airway problems in children can have a wide range of clinical consequences [6-8]. The main problems that are potentially related to UAO in patients with craniofacial dysostosis are failure to thrive, learning difficulties, 
and perioperative morbidity. These problems may contribute significantly to the overall disability of a child who may already have developmental impairment; and may represent an important complication of anaesthesia in patients who are likely to need a number of major surgical procedures.

In addition to the direct clinical consequences of UAO, it is well recognized in the adult population that OSA is associated with important cardiovascular changes, perturbations in blood gases, and changes in intrathoracic pressure [9]. It has been suggested that in craniofacial patients, with a high risk of intracranial hypertension, these factors may have important implications for intracranial blood flow/pressure dynamics. Thus, raised intracranial pressure and upper airway problems may be interrelated in these patients. Whilst there are a few anecdotal reports of such a relationship, to the best of our knowledge there are no formal studies in the current literature.

The aim of the present study was to make simultaneous recordings of ICP and respiratory signals, in order to examine the relationship between UAO and ICP during sleep, comparing a group of children with syndromic craniosynostosis with a control group of patients with isolated unicoronal stenosis only.

\section{Patients and methods}

\section{Patients}

We studied two groups of patients recruited from routine admissions to the multidisciplinary Craniofacial Unit at the Great Ormond Street Hospital between January 1992 and June 1994. We selected patients who were attending for their first craniofacial assessment, and who had not undergone any surgical procedure for the correction of the skull or facial morphology prior to the study. By including only patients who had not had corrective surgery, the number of eligible children was restricted to 20, from a total intake of approximately 125 patients over the period of the study.

Group 1 ("Syndromic" group). The syndromic group included 13 children ( 6 girls and 7 boys) with syndromic craniosynostosis, aged 3 months to 12 yrs (median $=9$ months). The diagnosis included six patients with Crouzon syndrome, five with Apert syndrome, one with Pfeiffer syndrome, and one with Saethre-Chotzen syndrome. These children all had both skull and facial abnormalities and were, therefore, anticipated to have some degree of respiratory involvement.

Group 2 ("Unicoronal" group). A control group of seven children ( 3 girls and 4 boys) with unicoronal synostosis, aged 3-29 months (median = 11 months). In these patients the deformities were restricted to the skull, with no facial involvement, and they were, therefore, not expected to have respiratory difficulties.

Patients were excluded from the study if they presented with any of the following: 1) known cardiac, neuromuscular or pulmonary disease; 2) upper airway infections in the preceding 3 weeks; 3) current medication with drugs causing muscle relaxation; 4) previous surgical procedure related to the craniofacial problems.
This research project was approved by the Institute of Child Health and Great Ormond Street Hospital Ethics Committee. In all cases, informed consent was obtained from the parents for ICP monitoring and sleep studies in their children.

\section{Protocol and methods}

The overall design of the study was to assess three different aspects of the children's disease: clinical history, physical examination and sleep study.

Clinical history. A detailed clinical history was taken, with special attention to the parents' impression of the nature, duration and severity of their child's respiratory problems. Based on the parents' descriptions, the degree of any respiratory problems was then classified as none, mild, moderate or severe, based on standard criteria used within our unit.

Physical examination. A physical examination was performed by an experienced clinician, prior to the performance of the sleep study (see below). The severity of the midfacial hypoplasia was judged on clinical appearance and was classified as normal, mild, moderate or severe.

Sleep study. A comprehensive sleep study was performed on each patient, with simultaneous monitoring of respiratory variables and continuous ICP.

On the morning preceding the study, a subdural intracranial pressure catheter was inserted through the skull, under inhaled general anaesthesia. This procedure is performed as part of the routine neurosurgical assessment of these patients in our unit. The procedure lasted approximately $15 \mathrm{~min}$ and involved the insertion of a subdural, miniature pressure transducer-tipped fibreoptic cable (OLM Intracranial Pressure Monitoring Catheter; San Diego, CA, USA). The catheter was connected to a dedicated monitor (Camino V420; San Diego, CA, USA) providing a continuous analogue output of the ICP waveform and a digital display of the mean intracranial pressure. Following recovery from the general anaesthetic, the patient was returned to the ward where ICP monitoring continued for the next $24 \mathrm{~h}$.

The following evening, and at least $8 \mathrm{~h}$ after general anaesthesia, a cardiorespiratory sleep study was performed during natural sleep, without the use of sedatives. Respiratory and ICP signals were recorded simultaneously on a dedicated paediatric sleep system comprising: IMS2000 Cardiorespiratory Sleep System (Pi Logic, Dyfed, UK) with PC-based Computer Aided Record Display and Analysis System (CARDAS) software (Oxford, UK). The IMS2000 system simultaneously recorded seven channels of data: 1) arterial oxygen saturation $\left(\mathrm{Sa}_{\mathrm{a}} \mathrm{O}_{2}\right)$; and 2) pulse rate (PR) from an in-built pulse-oximeter (Ohmeda Biox, Harlow, UK) sampling at a frequency of $1 \mathrm{~Hz}$; 3) rib cage (RC); and 4) abdominal (Ab) respiratory movements (uncalibrated strain-gauges; Mediplus, London, UK) sampled at $20 \mathrm{~Hz}$; 5) electrocardiogram (single-lead ECG) sampled at $100 \mathrm{~Hz} ; 6$ ) cardiac frequency $\left(f_{\mathrm{C}}\right)$ derived from the ECG, sampled at $1 \mathrm{~Hz}$; and 7) ICP from the indwelling probe (see above), sampled 
at $20 \mathrm{~Hz}$. The CARDAS software allowed the recording of all the variables in digital form, with subsequent semiautomated, interactive analysis of the data to allow review of the raw signals and calculation of summary statistics.

In addition to the computerized recording of polygraphic data, a video camera with directional microphone was used to make a permanent record of each sleep study. All sleep studies were monitored by the same experienced observer, who remained at the bedside throughout the studies making observations for sleep state analysis, and the detection and description of clinical signs of UAO. Sleep state analysis was based on behavioural criteria, according to PREChTL [10], with sleep state categorized into awake (W), quiet sleep (QS), active sleep (AS) and unknown (US) periods. The "unicoronal" group of patients was studied for periods sufficient to include at least two episodes both of QS and AS (i.e. a minimum of $2 \mathrm{~h}$ sleep); the "syndromic" patients tended to have more disrupted sleep states and had full overnight studies (up to $12 \mathrm{~h}$ ).

\section{Data analysis}

Sleep studies were subsequently analysed by interactive analysis with the computer, using the CARDAS software. Episodes of artefactual data (e.g. arterial oxygen saturation $\left(\mathrm{Sa}_{\mathrm{a}} \mathrm{O}_{2}\right)$ dips associated with movements or poor pulse waveform) were rejected manually, with verification from the video record.

Respiratory analysis. Respiratory signals were analysed in 1 min epochs throughout sleep for each sleep study. Inspection of the rib cage and abdominal respiratory movements provided the following information: 1) presence and absence of respiratory movements (detection of apnoeas); 2) the relative contribution both of rib cage and abdominal respiratory efforts to ventilation during the different sleep states; 3 ) the degree of synchrony or asynchrony between rib cage and abdominal movements; and 4) the presence of obstructive breathing patterns (alternating periods of high respiratory effort with poor ventilation and declining saturation, followed by arousal with a group of effective breaths and restoration of saturation).

The degree of UAO was classified as "mild", "moderate" or "severe", on the basis of the data from the sleep study combined with direct clinical observations of the patient's breathing pattern during sleep (table 1). Central apnoeas were defined as periods of absence of respiratory movements in excess of $6 \mathrm{~s}$, associated with a fall in $\mathrm{Sa}_{\mathrm{a}} \mathrm{O}_{2}$ and/or slowing of $f_{\mathrm{C}}$, and were identified by interactive analysis of the sleep study data and video record.

ICP analysis. For each individual, mean ICP levels were calculated for each sleep state (AS and QS). Inspection of the ICP tracings provided information on the pattern of the ICP waveform, and the relationship of alterations in this to changes in sleep state and respiratory pattern.
Table 1. - Criteria used for scoring the severity of upper airway obstruction during sleep

\section{Mild}

1. Clinical observation: slightly increased respiratory efforts, usually mouth breathing, \pm slight intercostal or suprasternal inspiratory recession, \pm snoring, without restless sleep

2. Sleep study: rib cage and abdominal signals in phase or slightly out of phase; $\mathrm{Sa}_{\mathrm{a}} \mathrm{O}_{2}$ within normal limits; \pm few brief, mild dips in $\mathrm{S}_{\mathrm{a}, \mathrm{O}_{2}}$, never $<90 \%$

\section{Moderate}

1. Clinical observation: moderately increased respiratory efforts, usually mouth breathing, \pm moderate intercostal or suprasternal inspiratory recession, \pm "snoring" and "snorting" with disrupted, restless sleep

2. Sleep study: rib cage and abdominal signals out of phase; baseline $\mathrm{Sa}_{\mathrm{a}} \mathrm{O}_{2}$ within normal limits; more frequent, repeated periods of desaturation to mid- 80 s\%

Severe

1. Clinical observation: markedly increased respiratory efforts, mouth breathing, marked intercostal or suprasternal inspiratory indrawings, \pm nasal flaring; loud "snoring" and "snorting" with disrupted, restless sleep

2. Sleep study: rib cage and abdominal signals out of phase, with frequent prolonged episodes of paradoxical breathing; frequent. prolonged periods of arterial oxygen desaturation

${\mathrm{Sa}, \mathrm{O}_{2}}_{2}$ arterial oxygen saturation.

\section{Statistical analysis}

The percentage concordance $(95 \%$ confidence interval $(95 \% \mathrm{CI})$ ) between the score for respiratory problems from clinical history (none/mild/moderate/severe) and sleep study UAO score (normal/mild/moderate/ severe) was calculated for the "syndromic" and "unicoronal" groups. Likewise, the percentage concordance $(95 \% \mathrm{CI})$ between the physical examination score for midfacial deformity (normal/mild/moderate/severe) and sleep study UAO score were calculated for both groups.

Mean ICP data for each individual were plotted on a scatter plot, with mean ICP during active sleep (AS) against mean ICP for quiet sleep (QS). A linear regression model (Minitab-Release 8) was used to relate mean ICP (AS) as the dependent term against mean ICP (QS) as the primary independent term. Disease severity was included as a second independent term, with the dummy variables of zero ("unicoronal") and one ("syndromic").

The nonparametric Spearman's rank correlation coefficients $\left(r_{\mathrm{s}}\right)$ between mean ICP and the sleep study score for the degree of upper airway obstruction (none/mild/ moderate/severe) both in QS and AS were calculated for all the patients, including both the unicoronal and the syndromic groups.

\section{Results}

Studies were successfully completed in all subjects, with at least $2 \mathrm{~h}$ of natural sleep available for analysis in each case (unicoronal group 2-4.5 h; syndromic: 4.5-12 h). A summary of the results both for the "syndromic" and the "unicoronal" patient groups is presented in table 2. 
Table 2. - Summary of individual results for sleep-related breathing disorders and ICP

\begin{tabular}{|c|c|c|c|c|c|c|c|c|c|c|c|c|c|}
\hline \multirow[t]{2}{*}{$\begin{array}{l}\mathrm{Pt} \\
\text { No. }\end{array}$} & \multirow[t]{2}{*}{$\begin{array}{l}\text { Age } \\
\text { yrs }\end{array}$} & \multirow[t]{2}{*}{ Sex } & \multirow[t]{2}{*}{ Diagnosis } & \multirow[t]{2}{*}{$\begin{array}{c}\text { Parents' } \\
\text { score }\end{array}$} & \multirow[t]{2}{*}{$\begin{array}{l}\text { Midface } \\
\text { exam }\end{array}$} & \multicolumn{2}{|c|}{$\begin{array}{l}\text { Respiratory } \\
\text { problems } \\
\text { seen in } \\
\text { sleep study }\end{array}$} & \multicolumn{2}{|c|}{$\begin{array}{c}\text { Mean } S \mathrm{a}, \mathrm{O}_{2} \\
\%\end{array}$} & \multicolumn{2}{|c|}{$\begin{array}{c}\text { Mean ICP } \\
\text { mmHg }\end{array}$} & \multicolumn{2}{|c|}{$\begin{array}{c}\text { Mean } f_{\mathrm{C}} \\
\text { bpm }\end{array}$} \\
\hline & & & & & & QS & AS & QS & AS & QS & AS & QS & AS \\
\hline \multicolumn{14}{|c|}{ Unicoronal patients } \\
\hline UI & 1.2 & M & & 0 & + & 0 & 0 & $98 \pm 0.5$ & $98 \pm 1.2$ & $9 \pm 2.0$ & $13 \pm 4.6$ & $94 \pm 7.1$ & $104 \pm 12.2$ \\
\hline U2 & 2.4 & $\mathrm{~F}$ & & 0 & 0 & 0 & 0 & $97 \pm 0.6$ & $97 \pm 0.8$ & $5 \pm 1.8$ & $5 \pm 1.5$ & $89 \pm 6.8$ & $87 \pm 9.0$ \\
\hline U3 & 0.3 & M & & 0 & 0 & 0 & 0 & - & - & $8 \pm 1.5$ & $11 \pm 3.6$ & $118 \pm 6.6$ & $129 \pm 8.9$ \\
\hline U4 & 1.0 & M & & 0 & 0 & 0 & 0 & $97 \pm 1.8$ & $97 \pm 2.8$ & $10 \pm 2.0$ & $17 \pm 4.3$ & $102 \pm 5.4$ & $114 \pm 7.7$ \\
\hline U5 & 0.3 & M & & 0 & 0 & 0 & 0 & $99 \pm 1.4$ & $97 \pm 3.6$ & $8 \pm 2.5$ & $9 \pm 3.2$ & $120 \pm 9.2$ & $126 \pm 12.0$ \\
\hline U6 & 0.9 & $\mathrm{~F}$ & & 0 & 0 & 0 & 0 & $98 \pm 0.8$ & $98 \pm 2.6$ & $14 \pm 2.6$ & $20 \pm 5.9$ & $114 \pm 4.3$ & $128 \pm 9.7$ \\
\hline U7 & 0.5 & $\mathrm{~F}$ & & 0 & 0 & 0 & 0 & $99 \pm 0.5$ & $99 \pm 2.0$ & $13 \pm 3.1$ & $17 \pm 5.4$ & $105 \pm 7.5$ & $109 \pm 7.8$ \\
\hline Mea & $\begin{array}{r}\text { n } 0.94 \\
\pm 0.73\end{array}$ & & & & & & & $98.0 \pm 0.9$ & $97.7 \pm 0.8$ & $9.6 \pm 3.1$ & $13.1 \pm 5.2$ & $106.0 \pm 11.9$ & $113.9 \pm 15.4$ \\
\hline \multicolumn{14}{|c|}{ Syndrome patients } \\
\hline S1 & 0.3 & $\mathrm{M}$ & Pfeiffer & 0 & + & 0 & 0 & $98 \pm 1.4$ & $97 \pm 2.6$ & $9 \pm 1.4$ & $11 \pm 2.6$ & $118 \pm 4.0$ & $124 \pm 6.7$ \\
\hline $\mathrm{S} 2$ & 1.5 & M & S-Chotzen & 0 & + & 0 & 0 & $95 \pm 0.6$ & $96 \pm 0.6$ & $24 \pm 1.9$ & $28 \pm 4.0$ & $107 \pm 5.9$ & $112 \pm 6.5$ \\
\hline S3 & 0.5 & M & Apert & 0 & ++ & + & $+^{*}$ & $96 \pm 0.6$ & $96 \pm 1.2$ & $8 \pm 1.9$ & $13 \pm 4.1$ & $124 \pm 6.8$ & $127 \pm 7.3$ \\
\hline S4 & 3.9 & M & Crouzon & 0 & + & + & $+^{*}$ & $97 \pm 0.9$ & $97 \pm 1.0$ & $11 \pm 2.7$ & $18 \pm 3.7$ & $93 \pm 7.1$ & $95 \pm 7.4$ \\
\hline S5 & 12.0 & $\mathrm{~F}$ & Crouzon & 0 & + & + & $+^{*}$ & $97 \pm 0.4$ & $97 \pm 0.7$ & $29 \pm 6.5$ & $42 \pm 6.7$ & $126 \pm 3.9$ & $129 \pm 5.9$ \\
\hline S6 & 2.3 & $\mathrm{~F}$ & Crouzon & + & ++ & + & ++ & $98 \pm 0.7$ & $98 \pm 0.9$ & $14 \pm 7.6$ & $30 \pm 7.8$ & $122 \pm 4.8$ & $125 \pm 6.1$ \\
\hline S7 & 0.4 & $\mathrm{~F}$ & Apert & +++ & +++ & ++ & $++^{*}$ & $98 \pm 1.0$ & $97 \pm 1.3$ & $8 \pm 3.0$ & $17 \pm 5.7$ & $128 \pm 6.5$ & $138 \pm 6.8$ \\
\hline S8 & 0.5 & $\mathrm{~F}$ & Crouzon & ++ & +++ & ++ & $++^{*}$ & $95 \pm 1.0$ & $95 \pm 1.9$ & $13 \pm 4.7$ & $22 \pm 10.9$ & $129 \pm 4.6$ & $133 \pm 7.8$ \\
\hline S9 & 0.3 & M & Crouzon & 0 & + & ++ & $++^{*}$ & $98 \pm 0.6$ & $98 \pm 1.4$ & $10 \pm 2.0$ & $18 \pm 7.4$ & $117 \pm 8.5$ & $125 \pm 11.6$ \\
\hline S10 & 3.1 & M & Crouzon & ++ & ++ & ++ & +++ & $93 \pm 1.2$ & $92 \pm 2.3$ & $18 \pm 5.0$ & $34 \pm 11.7$ & $101 \pm 5.0$ & $111 \pm 11.7$ \\
\hline S11 & 1.2 & $\mathrm{~F}$ & Apert & +++ & +++ & ++ & +++ & $94 \pm 0.9$ & $93 \pm 4.1$ & $20 \pm 4.2$ & $32 \pm 9.5$ & $130 \pm 4.0$ & $132 \pm 8.0$ \\
\hline $\mathrm{S} 12$ & 0.3 & $\mathrm{~F}$ & Apert & ++ & +++ & +++ & $+++*$ & $94 \pm 2.1$ & $93 \pm 3.9$ & $10 \pm 3.2$ & $12 \pm 4.1$ & $125 \pm 8.5$ & $130 \pm 12.6$ \\
\hline S13 & 0.8 & M & Apert & + & +++ & +++ & $+++*$ & $98 \pm 0.9$ & $94 \pm 4.5$ & $11 \pm 3.2$ & $21 \pm 6.3$ & $108 \pm 6.1$ & $111 \pm 11.7$ \\
\hline Mea & $\begin{array}{r}2.08 \\
\pm 3.20\end{array}$ & & & & & & & $96.2 \pm 1.8$ & $95.6 \pm 2.0$ & $14.2 \pm 6.6$ & $22.9 \pm 9.5$ & $117.5 \pm 11.8$ & $122.5 \pm 11.9$ \\
\hline
\end{tabular}

Values are presented as mean \pm SD. Parents' score: severity of respiratory problems base on the clinical history taken prior to the sleep study; Midface exam: score of severity of midfacial hypoplasia based upon a physical examination; Respiratory problems seen in sleep study: score of severity of upper airway obstruction based upon sleep study $(0=$ normal; $+=$ mild; $++=$ moderate; $+++=$ severe). *: further deterioration in active sleep, although still the same score as quiet sleep; $S_{\mathrm{a}, \mathrm{O}_{2}}$ : arterial oxygen saturation; ICP: intracranial pressure; $f_{\mathrm{C}}$ : cardiac frequency; QS: quiet sleep state; AS: active sleep state; M: male; F: female; Pt: patient; bpm: beats per minute; S-Chotzen: Saethre-Chotzen.

\section{Respiratory problems}

Clinical history. None of the "unicoronal" synostosis patients were reported by the parents as having any respiratory problems. In contrast, 7 of the 13 syndromic children were described as having various degrees of breathing difficulties, which ranged from mild to severe (table 2).

Physical examination. A mild midfacial hypoplasia was described in only one of the unicoronal group (No. U1), who had asymmetry of the orbits; in all other cases, the face was considered normal. Clinical assessment revealed some degree of hypoplasia in all of the syndromic patients, varying from mild to severe (table 2).

Sleep studies. Sleep state data, $S_{\mathrm{a}, \mathrm{O}_{2}}, f_{\mathrm{C}}, \mathrm{ICP}$ and the overall sleep study results are summarized in table 2 , whilst the signals from 2 representative sleep studies are shown in figure 1 . These studies illustrate the pattern of change with sleep state both in respiratory and ICP signals, for one "unicoronal" patient with normal breathing throughout sleep (fig. 1a) and one "syndromic" patient with severe OSA (fig. 1b). All seven unicoronal synostosis patients had normal breathing patterns during sleep. This was in marked contrast to the pattern observed in the group of "syndromic" children, in which
11 of the 13 patients had obstructive problems, which ranged from mild to severe (table 2). In the 11 syndromic patients with respiratory problems, UAO was always more severe during AS compared to QS (e.g. fig. $1 \mathrm{~b}$ and table 2). During active sleep, 8 out of 11 children either had moderate or severe obstruction. For the 11 patients with UAO, the mean $\mathrm{Sa}_{\mathrm{a}, \mathrm{O}_{2}}$ was $96 \%$ (SD $1.9 \%$ ) in quiet sleep and $95 \%$ (SD 2.2\%) in active sleep. In addition, $f_{C}$ tended to be higher in those with obstructive breathing, when compared to unicoronal patients of a similar age.

All the respiratory anomalies found were of the obstructive type, with no central respiratory abnormalities in any patient.

Concordance between clinical history, physical examination and sleep study scores for respiratory problems during sleep (table 2). For the "unicoronal" group of patients, there was a $100 \%$ concordance between the clinical history score, and the objective sleep study score for respiratory problems; in all instances, the children were scored as normal. For the comparison of physical examination scores, and sleep study scores, there was also perfect agreement, with the exception of one individual who was scored as having a mild midfacial hypoplasia, but in whom there was no evidence of respiratory problems during the sleep study. 
a) $2.5 \mathrm{~h}$ of sleep
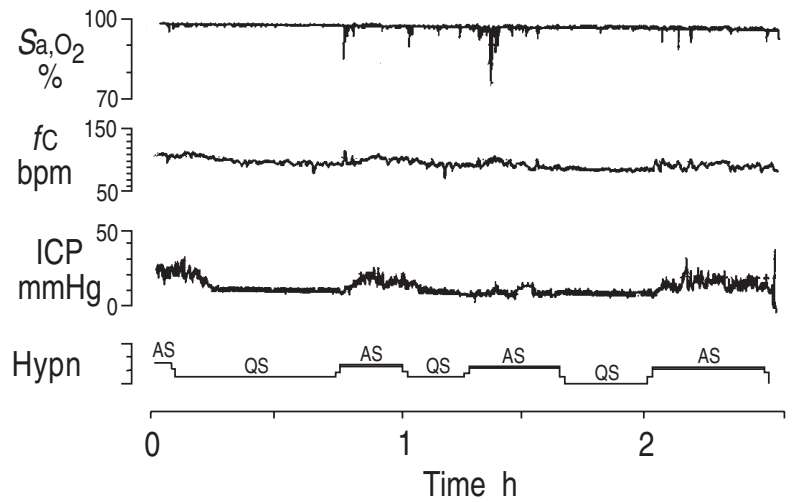

1 min of quiet sleep

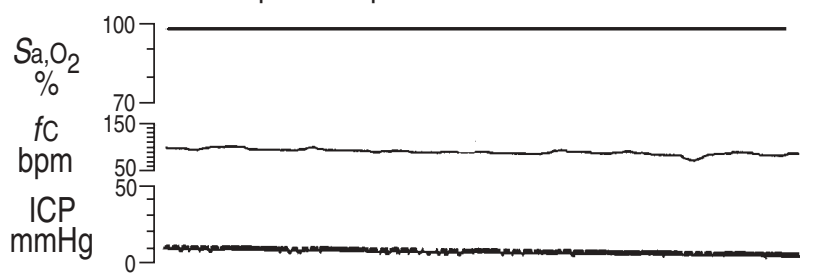

$\mathrm{RC}$

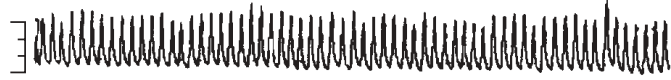

$\mathrm{Ab}$

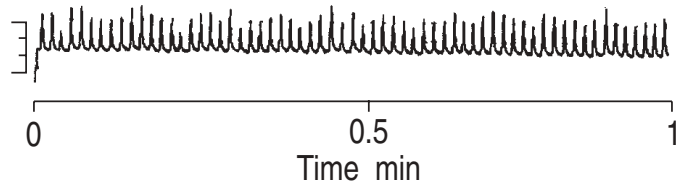

1 min of active sleep

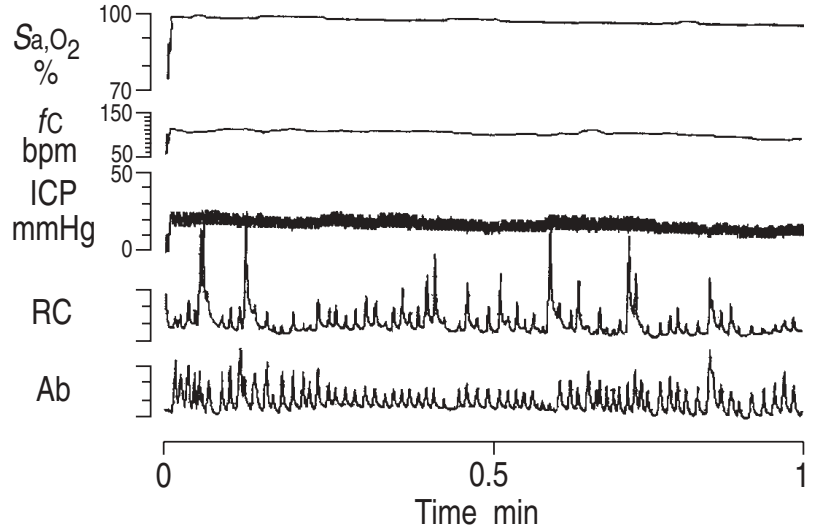

b) $\quad 2.5 \mathrm{~h}$ of sleep
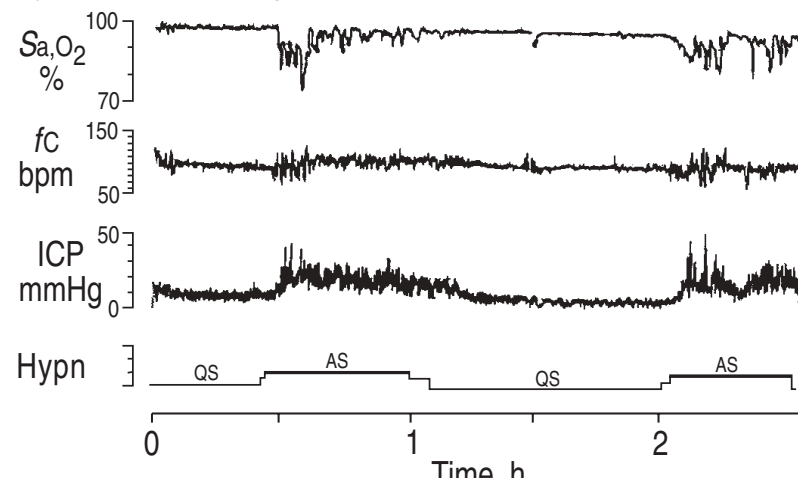

$1 \mathrm{~min}$ of quiet sleep

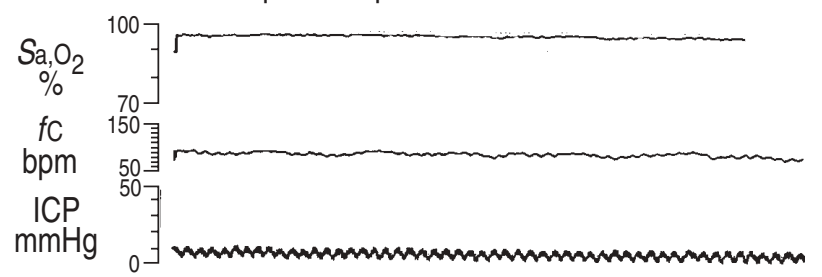

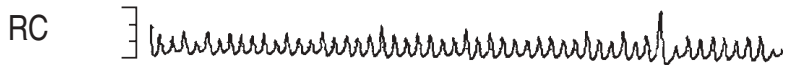

$\mathrm{Ab}$

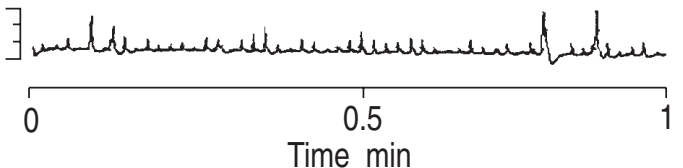

1 min of active sleep

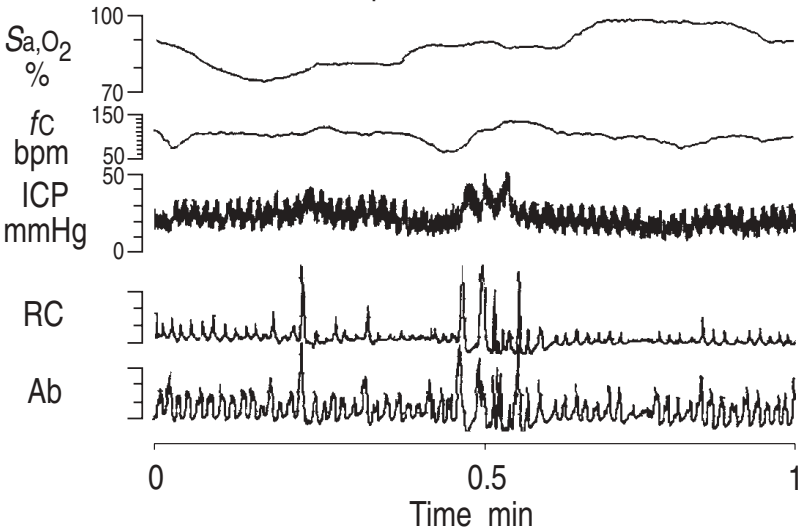

Fig. 1. - Sleep studies for two representative patients for: a) a unicoronal patient (U7) with normal respiratory sleep study; and b) a syndromic patient (S13) with severe upper airway obstruction, during $2.5 \mathrm{~h}$ of sleep, $1 \mathrm{~min}$ of quiet sleep and $1 \mathrm{~min}$ of active sleep. Sa, $\mathrm{O}_{2}$ : arterial oxygen saturation; $f_{C}$ : cardiac frequency; bpm: beats per minute; ICP: intracranial pressure; Hypn: hypnogram for sleep state; AS: active sleep; QS: quiet sleep; RC: rib cage respiratory movements; Ab: abdominal respiratory movements.

For the "syndromic" group, there was concordance in 4 out of 13 (31\%; 95\% CI 9-61\%) cases between clinical history score and objective sleep study score of UAO based upon the objective sleep study score of UAO for AS. In 8 out of $13(62 \%)$ cases, the parents underestimated the severity of the respiratory problems by at least one category; in only one case did the clinical history score exceed the objective sleep study score of UAO for AS. There was concordance between physical examination score and sleep study score in 6 out of 13 (46\%; 95\% CI 19-75\%) cases; physical examination scores overestimated the problem in 5 out of $13(38 \%)$ cases, whilst underestimating it in 2 out of $13(15 \%)$ cases.

\section{ICP Patterns}

In all patients, the pattern of ICP showed a relatively stable baseline during periods of quiet sleep, alternating with periods of more irregular, elevated ICP during active sleep, except for one "unicoronal" patient (No. U2), in whom mean ICP was the same for QS and AS, although 
the ICP signal was still more variable in AS. However, the variability of ICP in AS was smaller in the "unicoronal" than in the "syndromic" patients, in whom the amplitude of the ICP waveform tended to increase during AS (fig. 1). The mean (SD) values for ICP for individual patients, both during quiet and active sleep, are shown in table 2. Both "unicoronal" and "syndromic" groups showed a consistent increase in the ICP between QS and AS. Linear regression analysis of ICP(AS) against ICP $(Q S)$ showed a significant relationship between the two, with significant contributions from the independent variable of disease severity (unicoronal/syndromic), and the interactive term (ICP(QS) and disease severity). The calculated regression equation was:

$\mathrm{ICP}(\mathrm{AS})=1.32 \times \mathrm{ICP}(\mathrm{QS})+3.63 \times$ disease severity +0.52

This relationship indicates that mean ICP in active sleep (AS) is related by a factor of 1.32 (95\% CI 1.00$1.64 ; \mathrm{p}<0.001)$ to mean ICP(QS), and by a factor of $3.63(95 \%$ CI $-0.22-7.49 ; \mathrm{p}=0.076)$ to disease severity (i.e. if the patient is syndromic). In addition, $\mathrm{ICP}(\mathrm{AS})$ is increased on average by a constant of $0.52 \mathrm{mmHg}(95 \%$ CI -3.64-4.68; p=0.81) over mean ICP in quiet sleep (QS); however, this is not statistically significant.

\section{Relationship between ICP and respiratory patterns}

When analysed individually, both UAO and elevated ICP were more marked during active sleep, particularly in the "syndromic" patients. In children with UAO, inspection of simultaneous ICP and respiratory tracings shows a close association during AS (e.g. fig. 1b). Detailed analysis of short periods of active sleep, when individual breaths could be seen, showed that ICP was more constant in the unicoronal patients, in whom breathing patterns were normal (e.g. fig. 1a), compared with the more variable ICP pattern seen in the "syndromic" patients, in whom there was marked obstruction with irregular breathing patterns (e.g. fig. 1b).

During quiet sleep, all the unicoronal patients had normal breathing patterns and mean ICP values below $15 \mathrm{mmHg}$ (group mean ICP in QS $=9.6 \mathrm{mmHg}$; $95 \%$ CI 6.7-12.5 $\mathrm{mmHg}$ ). The group mean level of ICP in AS was $13.1 \mathrm{mmHg}(95 \% \mathrm{CI} 8.3-17.9 \mathrm{mmHg})$, with three patients having a mean ICP above $15 \mathrm{mmHg}$. The group mean elevation in ICP between quiet and active sleep was of $3.5 \mathrm{mmHg}$ (table 2).

In the syndromic group, there were 4 children with initially raised ICP in quiet sleep, and a total of 10 with levels above $15 \mathrm{mmHg}$ in active sleep. The group mean ICP in QS was $14.2 \mathrm{mmHg}$ (95\% CI 10.2-18.2 mmHg), whilst the group mean ICP in AS was $22.9 \mathrm{mmHg}(95 \%$ CI 17.2-28.6 mmHg). The mean increase in ICP between quiet and active sleep was $8.7 \mathrm{mmHg}$ (table 2).

Spearman's rank correlation for mean ICP versus sleep study UAO score (normal, mild, moderate or severe) for all patients studied was 0.36 ( $p>0.05)$ for quiet sleep and $0.55(0.05>\mathrm{p}>0.01)$ for active sleep.

\section{Discussion}

\section{Respiratory problems}

The principal finding of this study was that UAO comprised the only form of respiratory impairment in this group of children with craniosynostosis. In our sample of unoperated syndromic patients, $85 \%$ had some degree of UAO during sleep, with $61 \%$ having UAO that was thought to be clinically significant (moderate or severe on our scoring system), as they were associated with episodes of OSA. This contrasted markedly with the total absence of respiratory problems in the control group of "unicoronal" patients without facial involvement. Interestingly, despite appreciably raised ICP levels in many patients during AS, none showed any central respiratory abnormalities during sleep.

Clinically, it has been recognized that syndromic patients may have upper airway problems, which might be anticipated from the presence of obvious midfacial deformities. However, there is little objective documentation either on the extent or the severity of the respiratory problems in this group of patients [11-13]. In clinical practice, a major part of the assessment of upper airway function is based upon the clinical history taken from the parents. In the present study, we found that the respiratory problems tended to be underestimated by the parents; in two instances (Nos. S9 and S13) parents were unaware that their child had a degree of OSA that was, in fact, clinically important. This may be attributable to the complex nature of the condition of these patients, with other severe problems (e.g. raised ICP, cosmetic appearance, developmental delay) overshadowing the respiratory problems.

Assessment of the upper airways in these patients traditionally relies on physical examination of the airways and use of imaging techniques (e.g. radiography, computed tomography (CT), and magnetic resonance imaging (MRI)). However, these examinations are limited to descriptions of anatomical abnormalities, and provide no information about functional impairment. In addition, since the patient is commonly examined awake or sedated, the results may be misleading, as obstructive respiratory problems are state-related [7]. In the present study, the clinical assessment of the facial deformities by an experienced observer had reasonably good agreement with the findings of objective sleep studies. Nevertheless, in our experience, it is often impossible to evaluate the severity of sleep-related breathing problems in these patients without a sleep study.

Respiratory sleep studies allow the noninvasive assessment of upper airway function during different phases of natural sleep. It is important that unsedated sleep is assessed, as previous studies in these patients used sleep induced by barbiturates [14] and were devoid of rapid eye movement (REM) sleep (AS). Thus, upper airway problems and ICP levels were probably underestimated in these patients, as the relaxation of upper airway dilator muscles during REM sleep with increased likelihood of UAO, was not seen. The results of the present study confirm that the patients with UAO showed more severe respiratory problems during AS. In the eight children with moderate or severe UAO, the breathing pattern was typical of OSA, and dips in $\mathrm{Sa}, \mathrm{O}_{2}$ were deeper and more frequent.

For practical reasons, it was not possible to measure oronasal airflow in these patients; the children would not tolerate measurements made on or near the face, and it was necessary to exercise caution in order to avoid jeopardizing the clinically important ICP monitoring. 
Although it is not possible to distinguish between obstructive apnoeas and hypopnoeas in the absence of airflow measurements, it is still possible to identify the characteristic patterns of the obstructive cycle from the other measurements made. In obstructed patients, breathing was noisy, with snoring even in the mild cases, and loud snoring and snorting in more severe cases. The breathing patterns were cyclical, with periods of obstructive apnoea/hypopnoea interspersed with short periods of arousal (movement and increased cardiac frequency) and effective ventilation. Clinical observation identified periods of increased respiratory effort, often with paradoxical rib cage and abdominal movements, suprasternal/ intercostal indrawing, nasal flare and disrupted sleep.

Dips in $\mathrm{Sa}_{\mathrm{a}} \mathrm{O}_{2}$ are often used as a quantitative marker for the severity of upper airway obstruction. However, they should not be the only variable to be considered. In the present study, only 3 out of 8 children with moderate-to-severe obstruction had unsatisfactory mean $\mathrm{Sa}_{\mathrm{a}} \mathrm{O}_{2}$, with wide standard deviations (Nos. S10, S11 and S12). The mean $\mathrm{Sa}_{\mathrm{a}} \mathrm{O}_{2}$ tends to underestimate the severity of the obstructive problem, as the repetitive dips in $\mathrm{Sa}, \mathrm{O}_{2}$ alternating with possible overshoots during the recoveries tend to cancel out (e.g. patient No. S13, with severe obstruction (fig. 1a) had mean (SD) $\mathrm{Sa}_{\mathrm{a}} \mathrm{O}_{2}(\mathrm{QS})=98(0.9) \%$, $(\mathrm{AS})=94(4.5) \%) . S \mathrm{a}_{2} \mathrm{O}_{2}$ also failed to reflect the severity of the respiratory impairment in patient No. S9, who had adequate $\mathrm{Sa}_{\mathrm{a}} \mathrm{O}_{2}$ throughout the sleep study (mean 98\%) despite markedly increased breathing efforts. The amount of respiratory effort and the degree of sleep disturbance are probably important considerations when assessing the severity of sleep-related breathing disorders in these patients.

Secondary effects of OSA may have a number of important implications in craniofacial patients. Firstly, increased work of breathing against an obstructed upper airway may, in itself, be a sufficient consumer of energy to cause failure to thrive. Furthermore, disruption of sleep states in OSA, particularly loss of sustained QS, has been shown to impair growth hormone release [7, $15,16]$. Secondly, disturbed sleep patterns, with numerous arousals during the night, have been associated with poor daytime performance in children, with loss of concentration and learning difficulties $[7,15]$. Thirdly, upper airway problems are also associated with increased anaesthetic risk and increased perioperative morbidity [17, 18 , a problem which is particularly relevant to these patients.

\section{Intracranial pressure}

Our studies have shown that in patients with craniosynostosis ICP is not constant throughout sleep, but varies both within and between sleep states. Whilst it is possible to evaluate changes in ICP between sleep states, interpretation of absolute ICP values is difficult, as normal ranges for ICP in different age groups are yet to be established $[9,19,20]$.

The ICP waveform patterns of the present study were similar to those described in previous studies of patients with craniosynostosis $[21,22]$. In general, "syndromic" children have higher ICP levels than "unicoronal"; however, it has not been possible to predict the ICP level based on the number of sutures affected, nor the age of the patients. For both groups of patients, a consistent pattern of ICP changes during sleep was seen, with periods of relatively low baseline pressure during QS, alternating with periods of sustained elevated pressure during AS. In general, there was a rapid rise in ICP associated with the onset of AS, with a more gradual fall in ICP at the transition from AS to QS. The extent of the ICP increase during AS was related to the baseline ICP (QS). With a low baseline ICP in QS, relatively small elevations of the ICP plateaus were seen in AS, with low amplitude ICP oscillations, and a rapid return to baseline at the return to QS. In contrast, patients with high baseline ICP in QS had noticeable oscillations in ICP even during QS, with still higher ICP in AS, with large oscillations, and a slow return to baseline after the onset of the next QS phase.

The mechanisms behind the alterations in ICP levels between sleep states are multifactorial, probably involving metabolic, cardiovascular and respiratory effects. Slow wave sleep (QS) is associated with a decreased cerebral blood flow and glucose metabolism compared to REM sleep (AS) $[23,24]$. The elevated plateaus of ICP during AS in the present study probably correspond to the "A-waves" described by LundBERG [25], which are hypothesized to result from cerebrovascular dilation, and increased cerebral blood volume, combined with a poor volume/pressure relationship of the intracranial compartment [26]. Others have shown rises in ICP during REM sleep due to changes in cerebral blood flow $[27,28]$, and the amplitude of the ICP wave has been related to reduced cerebral compliance [22].

\section{Relationship between intracranial pressure and respi- ratory pattern}

To the best of our knowledge, the present study is the first to record both ICP and cardiorespiratory parameters simultaneously during natural sleep in children with craniosynostosis. A temporal relationship between raised ICP and breathing has previously been reported in adult neurosurgical patients with chronic intracranial hypertension and hydrocephalus [29], and with OSA [29, 30]. In adults with OSA, frequent episodic elevations of ICP have been observed in association with apnoeas [31]. Intracranial hypertension results from a number of different factors, including craniocerebral disproportion, abnormalities of cerebrospinal fluid (CSF) dynamics, impaired cerebral venous drainage, and hydrocephalus $[21,32]$. The present study indicates that ICP in AS is largely a function of the level of ICP during QS. However, the association of raised ICP plateaus with UAO during AS also appears to be important, and a number of physiological mechanisms can be proposed to relate the two.

Important changes in sleep physiology are seen as a consequence of the apnoeas during episodes of UAO while asleep [8]. In the craniofacial syndromes, skeletal deformities of the midface and skull base can result in anatomically distorted and narrowed upper airways. During sleep, particularly REM sleep, this situation is compounded by loss of tone in the upper airway dilator muscles [33]. With obstruction, there are increased 
inspiratory efforts against the closed airway, arterial carbon dioxide tension $\left(\mathrm{Pa}_{\mathrm{a}}, \mathrm{CO}_{2}\right)$ rises and arterial oxygen tension $\left(\mathrm{Pa}, \mathrm{O}_{2}\right)$ falls [8]. Both $f_{\mathrm{C}}$ and the blood pressure tend to fall $[34,35]$, and transient drops in intrathoracic pressure lead to reductions in aortic pressure and cardiac output (pulsus paradoxus) [36]. The combination of hypercapnia, hypoxia and increased inspiratory effort lead to arousal, which is associated with an increase in upper airway muscle tone, resumption of effective breathing, and acute rises in $f \mathrm{c}$, cardiac output and systemic blood pressure [35]. It may be postulated that the cycle of cardiovascular changes associated with OSA will have a direct effect on ICP through changes in arterial blood pressure and, thus, cerebral perfusion pressure.

There have been few clinical investigations on the interaction between ICP and OSA. SugiTA et al. [30] continuously monitored CSF pressure in the lumbar subarachnoid space in three adults with OSA. They observed episodic elevations of CSF pressure, always preceded and accompanied by episodes of apnoea or hypopnoea. The pressure regained normal levels within a few seconds after resumption of respiration. They suggested that transient increases in the intracranial blood volume (due to high $P \mathrm{a}_{1} \mathrm{CO}_{2}$ and low $\mathrm{Pa}, \mathrm{O}_{2}$ ) caused the rise in ICP.

Although we have been able to observe the coexistence of UAO and elevations in ICP in association with AS, the present study only allows us to speculate on possible causal mechanisms. On the one hand, it is possible that increased ICP may act directly, with brainstem compression contributing to obstructive respiratory problems [37]. On the other hand, OSA might indirectly contribute to increases in ICP, through changes in blood gas tension and elevations in blood pressure related to repeated arousals.

From a practical point of view, the main importance of UAO problems in craniosynostosis is probably related to the direct consequences of OSA on feeding, growth and development. However, respiratory problems may also constitute an important secondary cause for raised ICP. Further detailed studies are necessary to investigate the interrelationship between obstructive sleep apnoea and raised intracranial pressure and to define the physiological mechanisms involved. In terms of treatment, one might speculate that use of nasal continuous positive airway pressure (nCPAP), for example, may be successful in alleviating obstructive sleep apnoea and that this may also have a beneficial effect on intracranial pressure.

Acknowledgements: The authors gratefully acknowledge the Wellcome Trust for their funding of this work (Grant No. M/92/1817) and the "Conselho Nacional de Desenvolvimento Científico e Tecnologico" for supporting SG. They thank A. Wade, for statistical advice, and J. Stocks and D. Hatch for their help.

\section{References}

1. Marchac D, Renier D. Craniosynostosis. World J Surg 1989; 13: 358-365.

2. Shillito J, Matson DD. Craniosynostosis: a review of 519 surgical patients. Pediatrics 1968; 41 (4): 829-853.

3. Renier D. Intracranial pressure in craniosynostosis: preand postoperative recordings - correlation with functional results. In: Persing JA, Edgerton MT, Jane JA, eds. Scientific Foundations and Surgical Treatment of Craniosynostosis. Baltimore, Williams \& Wilkins, 1989: pp. 263-286.

4. James DR. Craniofacial anomalies. In: Evans J, ed. Paediatric Otolaryngology. 5th Edn. London, Butterworth \& Co. Ltd, 1987; pp. 226-275.

5. Mühlbauer W, Anderl H, Heeckt P, et al. Early operation in craniofacial dysostosis. World J Surg 1989; 13: 366-372.

6. Guilleminault C, Korobkin R, Winkle R. A review of 50 children with obstructive sleep apnea syndrome. Lung 1981; 159: 275-287.

7. Carroll J, Loughlin GM. Diagnostic criteria for obstructive sleep apnea syndrome in children (guest editorial). Pediatr Pulmonol 1992; 14: 71-74.

8. Sheldon SH, Spire J-P, Levy HB. Sleep-disordered respiration in childhood. In: Pediatric Sleep Medicine. 1st Edn. Philadelphia, W.B. Saunders Co, 1992; pp. 136-150.

9. Stradling JR. Pathophysiology of obstructive sleep apnoea. In: Handbook of Sleep-Related Breathing Disorders. 1st Edn. Oxford, Oxford University Press, 1993; pp. 23-64.

10. Prechtl HFR. The behavioural states of the newborn infant (a review). Brain Res 1974; 76: 185-212.

11. Edwards TJC, David DJ, Martin J. Aggressive surgical management of sleep apnoea syndrome in the syndromal craniosynostoses. In: Montoya AG, ed. Craniofacial Surgery 4. Proceedings of the 4th Meeting of the International Society of Cranio-maxillo-facial Surgery. Bologna, Monduzzi Editore, 1992; pp. 189-192.

12. Mixter RC, David DJ, Perloff WH, Green CG, Pauli RM, Popic PM. Obstructive sleep apnea in Apert's and Pfeiffer's syndromes: more than a craniofacial abnormality. Plast Reconstr Surg 1990; 86 (3): 457-463.

13. Lauritzen C, Lilja J, Jarlstedt J. Airway obstruction and sleep apnea in children with craniofacial anomalies. Plast Reconstr Surg 1986; 77 (1): 1-5.

14. Moore MH. Upper airway obstruction in the syndromal craniosynostosis. Br J Plast Surg 1993; 46: 355-362.

15. Stradling JR. Paediatric aspects of obstructive sleep apnoea. In: Handbook of Sleep-Related Breathing Disorders. 1st Edn. Oxford, Oxford University Press, 1993; 172-187.

16. Potsic WP. Obstructive sleep apnoea. Pediatr Clin North Am 1989; 36 (6): 1435-1442.

17. Helfaer MA, Wilson MD. Obstructive sleep apnoea, control of ventilation, and anesthesia in children. Pediatr Clin North Am 1994; 41 (1): 131-151.

18. Rosen GM, Muckle RP, Mahowald MW, Goding GS, Ullevig C. Postoperative respiratory compromise in children with obstructive sleep apnea syndrome: can it be anticipated? Pediatrics 1994; 93 (5): 784-788.

19. Minns RA. Problems of intracranial pressure in childhood. In: Minns RA, ed. Clinics in Developmental Medicine. Nos. 113-114. London, MacKieth Press, 1991.

20. Thompson D, Harkness W, Jones B, Gonsalez S, Andar U, Hayward R. Subdural intracranial pressure monitoring in craniosynostosis - its role in surgical management. Childs Nerv Sys 1995; 11: 269-275.

21. Whittle IR, Johnston IH, Besser M. Intracranial pressure changes in craniostenosis. Surg Neurol 1984; 21: 367-372.

22. Renier D, Sainte-Rose C, Marchac D, Hirsch J-F. Intracranial pressure in craniostenosis. J Neurosurg 1982; 57: 370-377.

23. Meyer JS, Ishikawa Y, Takashi H, Karacan I. Cerebral blood flow in normal and abnormal sleep and dreaming. Brain Cogn 1987; 6: 266-294. 
24. Fischer AQ, Taormina MA, Akhtar B, Chaudhary BA. The effect of sleep on intracranial hemodynamics: a transcranial doppler study. J Child Neurol 1991; 6: 155-158.

25. Lundberg N. Continuous recording and control of ventricular fluid pressure in neurosurgical practice. Acta Psychiatr 1960; 36 (149): 1-193.

26. Risberg J, Lundberg N, Ingvar DH. Regional cerebral blood volume during acute transient rises of the intracranial pressure (plateau waves). J Neurosurg 1969; 31: 303-310.

27. Munari C, Calbucci F. Correlations between intracranial pressure and EEG during coma and sleep. Electroencephalogr Clin Neurophysiol 1981; 51: 170-176.

28. Rossi GF, Maira G, Vignati A. Intracranial pressure during sleep in man. Sleep 1974. 2nd European Congress on Sleep Research, Rome 1974. Karger, Basel, 1975; pp. 169-180.

29. Yokota A, Matsuoka S, Ishikawa T, Kohshi K, Kajiwara H. Overnight recordings of intracranial pressure and electroencephalography in neurosurgical patients. Part II. Changes in intracranial pressure during sleep. Sangyo Ika Daigaku Zasshi 1989; 11 (4): 383-391.

30. Sugita Y, Iijima S, Teshima Y, et al. Marked episodic elevation of cerebrospinal fluid pressure during nocturnal sleep in patients with sleep apnea hypersomnia syndrome. Electroencephalogr Clin Neurophysiol 1985; 60: 214-219.
31. Fischer A, Chaudhary B, Taormina M, Akhtar B. Intracranial hemodynamics in sleep apnea. Chest 1992; 102 : $1402-1406$.

32. Thompson D, Jones B, Hayward R, Harkness W. Assessment and treatment of craniosynostosis. Br J Hosp Med 1994; 52 (1): 17-24.

33. Remmers JE, de Groot WJ, Sauerland EK, Anch AM. Pathogenesis of upper airway occlusion during sleep. $J$ Appl Physiol: Respirat Environ Exercise Physiol 1978; 44 (6): 931-938.

34. Andreas S, Hajak G, Breska B, Rüther E, Kreuzer H. Changes in heart rate during obstructive sleep apnoea. Eur Respir J 1992; 5: 853-857.

35. Bonsignore MR, Marrone O, Insalaco G, Bonsignore G. The cardiovascular effects of obstructive sleep apnoeas: analysis of pathogenic mechanisms. Eur Respir J 1994; 7: 786-805.

36. Shiomi T, Guilleminault C, Stoohs R, Schnittger I. Leftward shift of the interventricular septum and pulsus paradoxus in obstructive sleep apnea syndrome. Chest 1991; 100: 894-902.

37. Nickerson B. Control of breathing problems associated with Arnold-Chiari malformation. In: Beckerman RC, Brouillette RT \& Hunt CE, eds. Respiratory Control Disorders in Infants and Children. Baltimore, Williams \& Wilkins, 1992. 